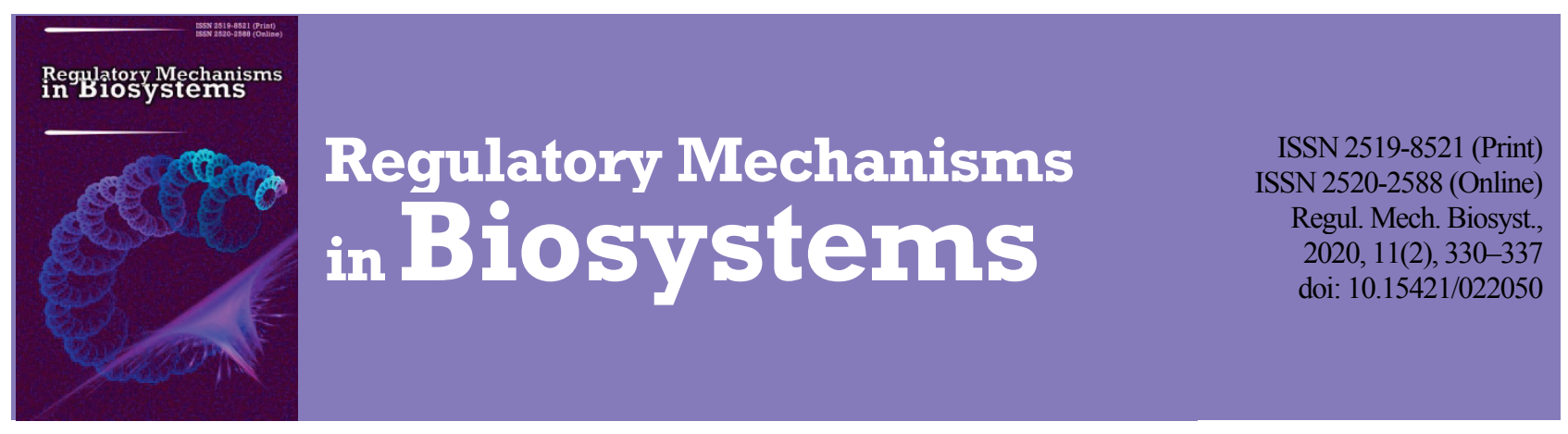

\title{
Morphological and radiological study of lymph nodes in dromedaries in Algeria
}

\author{
D. E. Rahmoun*, M. A. Lieshchova**, M. A. Fares* \\ *University of Mohamed Cherif Messaâdia, Souk Ahras, Algeria \\ **Dnipro State Agrarian and Economic University, Dnipro, Ukraine
}

Article info

Received 09.04.2020

Received in revised form 12.05.2020

Accepted 13.05.2020

University of Mohamed Cherif Messaâdia,

Souk Ahras, 41000, Algeria

Tel.: +213-663-121-367.

E-mail:

deddine44@hotmail.com

Dnipro State Agrarian and Economic University

Sergii Efremov st., 25 ,

Dnipro, 49600, Ukraine.

Tel.: +38-067-256-24-86

E-mail:

lieshchova.m.o@dsau.dp.ua

\begin{abstract}
Rahmoun, D. E., Lieshchova, M. A., \& Fares, M. A. (2020). Morphological and radiological study of lymph nodes in dromedaries in Algeria. Regulatory Mechanisms in Biosystems, 11(2), 330-337. doi:10.15421/022050

Despite significant progress in the study of the subtle mechanisms of interaction between cellular and molecular elements in immune responses, the general structure of the organs of the immune system, including the lymph node, has not been sufficiently studied, in particular in large farm animals. The lymph nodes of sexually mature camels have been studied anatomically and morphologically and advanced studies conducted using an X-ray system and a computer densitometer scanner with injection of a contrast medium. The topography and characteristics of the morphometric parameters (absolute and relative mass, linear measurements, volume) of certain somatic and visceral lymph nodes were determined. The mass of the lymph nodes studied varies according to the location and the interest of the organ in the satellite defense of the lymphoid system, For part of the x-ray examination of the lymph nodes, organs of large inguinal and axillary shape were selected after passing through a solution of tetraethyl-4,4-diamino-triphenylmethane oxalate, the lymph vessels were dilated and darkened, then iodine injections were made into the afferent lymphatic vessel of two lymph nodes; they were placed on the radiological cassette, a photograph taken on conventional radiography, for computer densitometer, the examination was made without preparation of the organs. A capsule encompasses the parenchyma of the lymph node, whose internal structure is composed of different zones, cortical, paracortical and medullary, on the one hand the lymphatic vessels were very clear especially with the conventional radiography with preparation of the organs, while the computer densitometer clearly revealed the deep texture of the parenchyma, basing it on the intensity of emission saturation from the use of computer densitometer.
\end{abstract}

Keywords: anatomy; topography; dromedary; morphometric parameters; radiology; contrast agent.

\section{Introduction}

Lymph nodes are peripheral organs of the lymphatic system (Rebelatto, 2018). They serve as a biological filter through which the lymph flows from the organs and parts of the body. Lymph nodes provide immunological protection by cleaning the lymph from pathogens and foreign agents (Willard-Mack, 2006). They also deposit lymph, perform an antigen-dependent proliferation and differentiate $\mathrm{T}$ and $\mathrm{B}$ lymphocytes into effector cells. In the lymph nodes, factors of cellular and humoral immunity are formed, the formation of memory cells is noted (Pabst, 2020). In the lymph node, the lymph circulates through a complex system of lymphatic sinuses: subcapsular, intermediate, cortical, medullar and chylous, where conditions are created for the interaction of antigens with cells presenting special antigens. Lymphoid cells also participate in lymphatic cleansing. Due to antigenic stimulation, proliferation of lymphocytes and plasma cells occurs and, therefore, antibodies enter the lymph (Elmore, 2006).

According to the generally accepted scheme for describing the structure of the lymph node, a framework of connective tissue is distinguished there, which includes a capsule with thickening of the hilum and trabeculae which leave it, as well as a lymphoid parenchyma formed by reticular tissue with cellular elements located within it. The lymph node capsule and trabeculae are made up of dense, unformed fibrous connective tissue with collagen, a small amount of elastic and reticular fibers, and smooth muscle cells, which are myocytes (Harisinghani, 2012). The trabeculae are connected to each other and form a spongy armour designed by the frame. According to Gavrilin et al. $(2015,2017 \mathrm{a}, 2017 \mathrm{~b})$ the skeleton of the intranodal connective tissue of the dromedary lymph node is represented by two types of trabeculae, we note the muscular connective tissue (type I) and the smooth muscle (type II). The lymphatic sinuses are divided into large sub capsular and peritrabecular trabeculae of type I, as well as less developed, for peritrabecular trabeculae of type II and medullar cord. An analysis of data from the literature on the development of active follicles in mammals in ontogenesis indicates that the laying of various groups of nodes does not take place simultaneously, and the timing of their laying depends on the location (Margaris \& Black, 2012). Lobular lymph node structure is formed in mammals during embryogenesis following the introduction of mesenchymal structures in the light of lymphatic sinuses (Mebius, 2003). The appearance of signs of a definitive structure of the lymph node parenchyma in mammals is debatable. In the work of various authors, it varies from the middle of the fetal period to the initial stages of postnatal ontogenesis (Taher et al., 1989). According to studies of the lymph nodes of fetuses from cattle, the isolation of lymphoid lobules and the formation of the complex of the main functional areas of the parenchyma occur from 5 months (Gavrilin et al., 2018).

The dromedary (Camelus dromedarius Linnaeus, 1758), is a species of mammal with the most advanced adaptation mechanisms to difficult climatic conditions. In this study on this species according to data already exploited by (Nourinezhad et al., 2015, 2016), we used several different lymph nodes; aspects of the dromedary lymph nodes are discussed. The position of the dromedary lymph nodes is summarized and illustrated in anatomical tables explaining the position and precise topography of each unit. Studies have been carried out on the macroscopic aspects of the lymph nodes in dromedaries (Abdel-Magied et al., 2001).

The number of lymph nodes in the dromedary is only about 131 (Fowler et al., 1988). Simple lymph nodes are located in connective tissue or adipose tissue and can be arranged in a chain or grouped in clusters. Their size is variable and depends on the age of the animal. In the works devoted to the structure of the lymph nodes, it is indicated that their parenchyma is divided into specialized zones, without any precise diagram of their relative position and their localization (Abdel-Magied et al., 2001; 
Zidan \& Pabst, 2002, 2012). According to the authors, it was found that the morphology and the lymph node structure of the dromedary have intermediate characters which are characteristic of both mammals in general and in particular of the pig (Gavrilin et al., 2017; Kraitchman et al., 2017). Detailed anatomical data must be obtained concerning the lymph nodes which play an important role in the defense mechanism of the body against the invasion of foreign bodies (Reiter et al., 2018). In addition, they can be easily felt in animals during meat inspection. Studies have been carried out on the macroscopic aspects of the lymph nodes in dromedaries, while there is a lack of data on the anatomy and the topography of the lymph nodes, especially for this particular animal.

This has allows us to know with certainty that the lymph node of the dromedary is complex. The lack of information means that a thorough exploration of these organs in this species is required, all the results that we could have had after the analysis are directly in line with the theory of immunology, such a complex requires work with conventional methods and modern, ultra structure is the problem of the day, according to the authors who have worked on the camel's immune system. The use of so-called "conventional" radiology is the exploration of internal anatomical structures using the image provided by a beam of X-rays passing through the organ (Maher et al., 2020). It remains relevant in the foreground exploitation in many fields of medicine in spite of the appearance of new techniques. According to the nature of the tissues, crossed X-rays will be more or less attenuated and will give in the end a radiological contrasted image. The identification by imaging of the normal lymphatic channels has always been a difficult exercise due to their very small size, their slow flow and the ignorance of their anatomy which was mentioned by the authors (Gor et al., 2006).

Imaging is rarely used routinely for the study of lymphatic circulation because it is complex to implement. Imaging of the lymphatic pathways is now mainly based on lymphography; the caliber of normal lymphatic pathways makes it possible to obtain images of these pathways (Kelch et al., 2019). The image of normal lymphatic pathways is not described because the lymphatic anatomy and radiology skills are poorly understood in veterinary medicine. There is almost no work in the world literature on this subject.

The use of imaging for precise identification and characterization of the lymph nodes are of major importance, so it becomes crucial to have information in this area to initiate research with imaging modalities in different sections, which currently rely on the morphological criteria of the organ. In recent years, studies have shown that these new imaging techniques can bridge some of the limitations of existing imaging for nodal characterization and thus provide valuable information for the first time. This will be used in dromedaries as a type animal hence the need to develop targeted techniques for nodal imaging and characterization as indicated in this article.

Our goal in this research is to evidence the macroscopic appearance of the lymph nodes of the dromedary and also deepen through the radiology technique knowledge of the in situ structure of this organ.

\section{Materials and methods}

The protocol of the experimental part of the study at the stages of animal care and their withdrawal from the experiment corresponded to the principles of biological ethics, the removal of the organs of which was done at the level of the meat slaughterhouses, for human consumption, agreed with the local ethics committee of Souk Ahras University, Algeria.

The somatic (inguinal, axillary, popliteal) and visceral (retro pharyngeal, tracheobronchial, mesenteric) lymph nodes studied were obtained at the slaughterhouse, from ten mature adult camels (Camelus dromedarius), of the Ouled Sidi Chikh breed from southern Algeria. They were clinically healthy and untreated preventively, the animals' weight was between 328 and $454 \mathrm{~kg}$. The organs were removed after dissection and anatomical evisceration of the animals.

During sampling, the anatomo-topographic characteristics of each lymph node were determined, the organs studied were prepared according to a specific process in which the fatty tissue that envelops these organs was carefully removed, and this step requires the use of a scalpel. Pictures of organs were taken with a digital camera Nikon D7500 and processing on PC. The absolute mass of each lymph node was adjusted using an electronic scale "Tehniprot-WTW", up to $0.002 \mathrm{mg}$, and also the relative mass of the organs to the body mass of the animal was calculated.

Linear dimensions (length, width) of each lymph node were determined using a centimeter ruler with a division of $1 \mathrm{~mm}$. The volume of each lymph node was calculated using a volumetric technique, in a container graduated in milliliters which contains a coloured solution, the organ was immersed and the difference in excess of the liquid estimated in $\mathrm{mm}^{3}$.

The radiological exploration of the lymph nodes was carried out in a diagnostic radiology clinic with the help of a radiology operator. Standard and conventional radiography was used with an AeroDR X60 Konica Minolta system, with a $360^{\circ}$ rotation. Screen 10 inch as a central interface for intelligent control and monitoring during the examination. The technique was carried out in two stages, the first step without preparation, i.e. there is no injection of contrast agent in the organ, whereas the second step required an injection of contrast agent Radioselectan UV 76\% 370 mg of Iodine / $\mathrm{mL}$ Meglumine amidotrizoate, with a very fine syringe needle, one dose $0.1 \mathrm{~mL}$ was injected through some lymphatic vessels of the inguinal and axillary lymph nodes after they were impregnated in a solution of tetra-ethyl-4,4-diamino triphenyl methane oxalate for 24 hours in order to reduce the rigidity of the organ and facilitate penetration of the contrast medium. This technique makes it possible to detect a linear harmonic response of the various structures of the organ and also a threedimensional view and it depended on the nature of the contrast product and its infiltration. The organs are placed on a cassette which contains an $\mathrm{X}$-ray sensitive fluorescent film, the images are developed in a dark room with an XR 24 PRO digital development device, the quality of the radiographic image conditions the interpretation of the radiographic organs, we observe black zones corresponding to air and corresponding white zone structures of the different compartments. We identify the contours of the organs, clearly distinguished and visible areas. This technique makes it possible to detect a linear harmonic response of the various structures of the organ with a three-dimensional view and it depends on the nature of the contrast product and its infiltration.

For scanning with a CT scanner, the device used was the CT-Tomography-16 Scanner-Scintcare CT 16-Scanner, the axillary and inguinal lymph nodes were immersed in a glass container of distilled water, we note that in this stage of examination, the organs had not undergone any prior preparation and were then placed on the cart of the CT scanner. The cuts were made on an apparatus with multiple bars MX 8000 (Marconi ${ }^{(B)}$ ) without injection of contrast in spiral acquisition in fused sections of 2 or $3 \mathrm{~mm}$ with reconstruction of fine axial and coronal sections in window for the soft parts, photographs were stored on the device monitoring screen, a treatment of different three-dimensional trajectories was made.

Statistical analysis of the results was carried out in Statistica 10.0 (StatSoft Inc., USA). Differences between samples were determined using ANOVA and were considered significant at $\mathrm{P}<0.05$.

\section{Results}

After dissection of the animal, the somatic lymph nodes were the first to be investigated, it was found that the axillary lymph node appears constant, more or less flattened of almost circular shape, it is placed at the level of the third rib on the serrated muscle of the thorax and below the axillary vein (Fig. 1). Its deep face is related to the large round muscle and the thoraco-dorsal artery. The popliteal lymph node appears constant and has an ovoid shape. And located in the popliteal fossa, it is almost on the surface, however hidden by the thick elastic blade which strengthens the leg fascia, for the inguinal lymph node, it appears bulky, enormous, elongated and spindle-shaped. This lymph node in the male is located in the crease of the groin a hand's breadth above the rudimentary teats. It remains on the abdominal coat. The scrotal node is related to the caudal preputial muscles and limited ventrally by the abdominal subcutaneous vein. Whereas in the female, it was located in the region of the inguinal. The two ganglia are side by side and are in the perimammary position. They are covered by the skin and are related to the pectineal muscles.

For the visceral lymph nodes, it was detected that the mesenteric lymph node is located in the terminal part of the rectum. The tracheobronchial lymph node is superimposed on the bifurcation of the trachea to- 
wards the bronchi, and it was found that this lymph node is composed of two symmetrical portions: the right and left tracheobronchial nodes. It has been observed that the right tracheobronchial lymph node is placed against the termination of the trachea at the birth of the tracheal bronchus. The left tracheobronchial lymph node was bifurcated with a thin flat part and the other spherical. It is visible on the left behind the aortic arch, for the retropharyngeal lymph node seems bulky and hollowed out by a groove where the common carotid artery lodges; it is in cranial connection with the mandibular lymph node and dorsally with the occipital artery (Fig. 2).

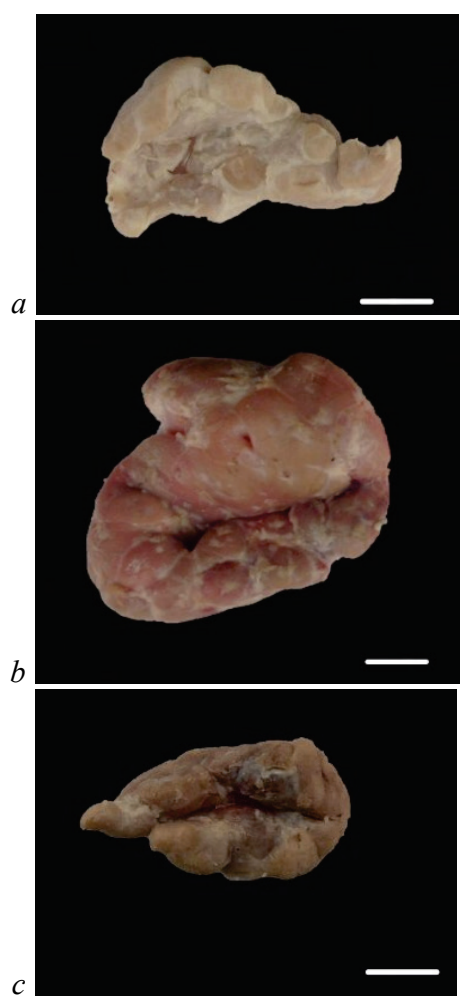

Fig. 1. The morphometry of certain somatic lymph nodes in dromedaries: $a$-inguinal lymph node, $b$-axillary lymph node, $c$-popliteal lymph node; bar $-1 \mathrm{~cm}$

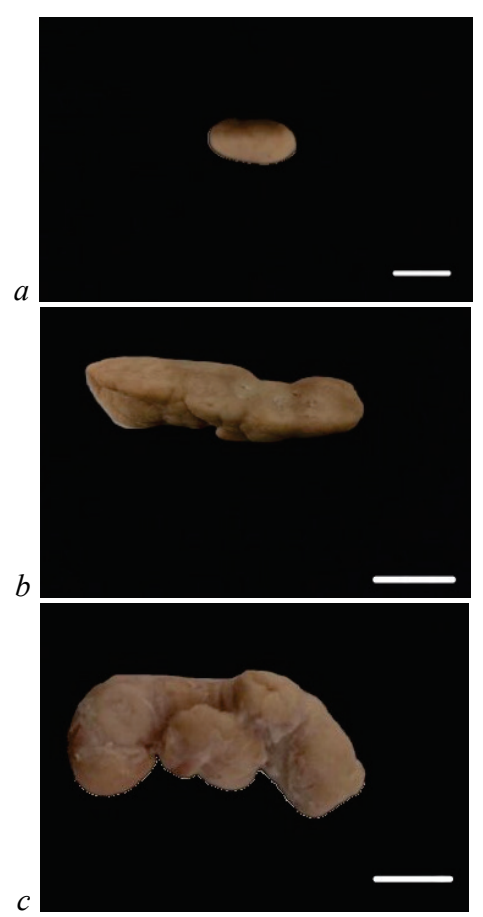

Fig. 2. The morphometry of certain visceral lymph nodes in dromedaries: $a$-tracheobronchial lymph node, $b$ - retropharyngeal lymph node, $c$-mesenteric lymph node; bar $-1 \mathrm{~cm}$
After measuring the mass of the sampled lymph nodes, and calculating the relative mass of each one with respect to the weight of the animal, it was found that the maximum value of the absolute mass was $42.75 \mathrm{~g}$ found on the somatic lymph node axillary, with a relative mass of $0.012 \%$, and the minimum value of absolute mass was $4.23 \mathrm{~g}$ found on the visceral tracheobronchial lymph node, with a relative mass of $0.006 \%$ (Table 1 ).

Table

Absolute and relative mass of the lymph nodes in dromedaries

\begin{tabular}{clcc}
\hline & Lymph nodes & Absolute mass, $\mathrm{g}$ & Relative mass, \% \\
\hline \multirow{2}{*}{ Somatic } & inguinal & $11.51 \pm 0.145$ & $0.003 \pm 0.0005$ \\
& axillary & $42.75 \pm 0.149$ & $0.012 \pm 0.0002$ \\
& popliteal & $17.42 \pm 0.125$ & $0.005 \pm 0.0002$ \\
\hline \multirow{2}{*}{ Visceral } & retropharyngeal & $15.72 \pm 0.158$ & $0.008 \pm 0.0005$ \\
& tracheal bronchial & $4.23 \pm 0.149$ & $0.006 \pm 0.0009$ \\
& mesenteric & $7.04 \pm 0.125$ & $0.004 \pm 0.0007$ \\
\hline
\end{tabular}

After measuring the length and width of the somatic lymph nodes (popliteal, inguinal and axillary), it was found that the maximum value of the length was $8.20 \mathrm{~cm}$ for the axillary lymph node with a width of $6.90 \pm$ $0.694 \mathrm{~cm}$, for the inguinal lymph node, the minimum length was $5.30 \mathrm{~cm}$ with a width of $2.60 \pm 0.126 \mathrm{~cm}$, for the popliteal somatic lymph node, its length was $6.70 \pm 0.129 \mathrm{~cm}$ and a width of $3.50 \pm 0.147 \mathrm{~cm}$, taking pictures of each lymph node helped us a lot in studying the external morphology of this organ (Fig. 3).
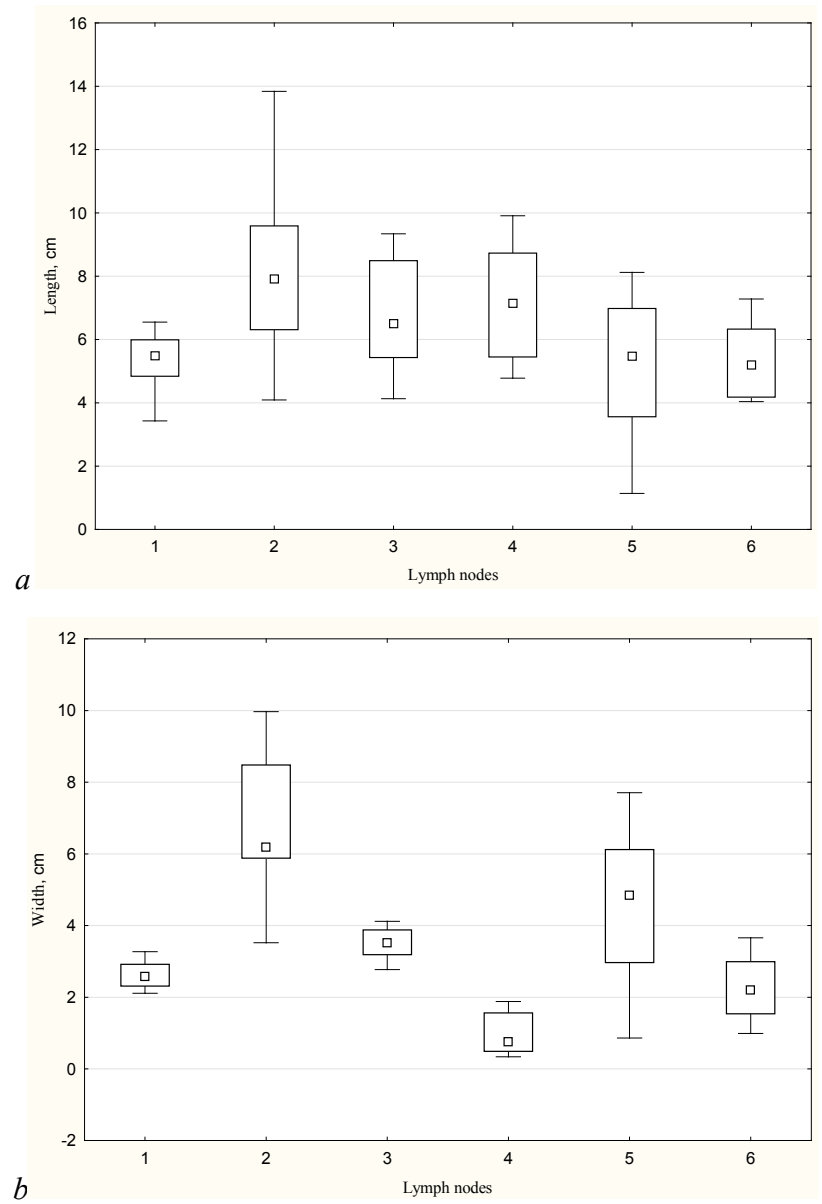

Fig. 3. Length $(a)$ and width $(b)$ of certain lymph nodes in dromedaries $(\mathrm{n}=10): 1$-inguinal lymph node, 2 - axillary lymph node, 3 - popliteal lymph node, 4 -retropharyngeal lymph node, 5 - tracheobronchial lymph node, 6 -mesenteric lymph node

Concerning the visceral lymph nodes, the retropharyngeal presented a maximum length of $7.20 \pm 0.114 \mathrm{~cm}$ and a width of $3.90 \pm 0.192 \mathrm{~cm}$, while the minimum length for the tracheobronchial lymph node was $3.90 \pm$ $0.756 \mathrm{~cm}$ and a width of $1.30 \pm 0.745 \mathrm{~cm}$, and the mesenteric lymph node had a length of $5.40 \pm 0.385 \mathrm{~cm}$ with a width of $2.30 \pm 0.296 \mathrm{~cm}$ (Fig. 3). After measuring the volume of the lymph nodes, it was found that the 
maximum value of the volume was $0.439 \mathrm{~mm}^{3}$ in the axillary somatic lymph node with relative volume of $0.605 \%$, and the minimum value was $0.028 \mathrm{~mm}^{3}$, tracheobronchial visceral lymph node with relative volume of $0.079 \%$. And the other results of volume are mentioned in Figure 4.

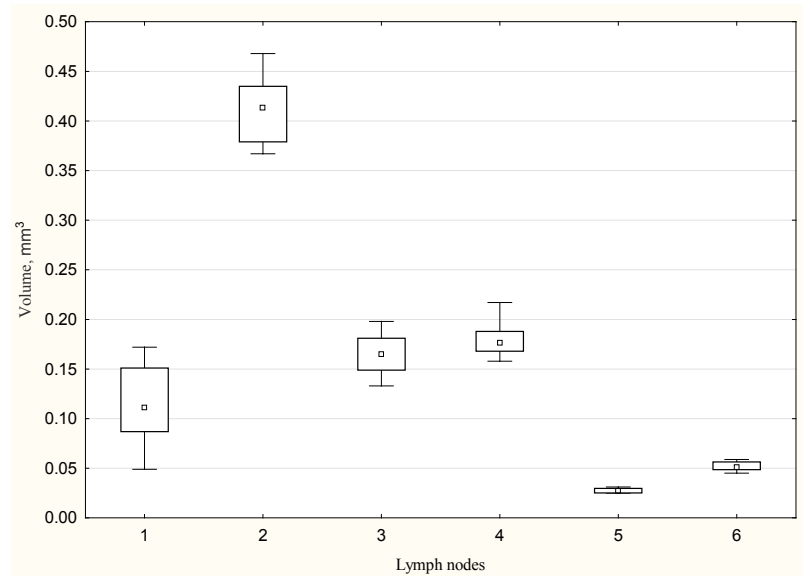

Fig. 4. Volume of certain lymph nodes in dromedaries $(n=10)$ : 1 -inguinal lymph node, 2 - axillary lymph node, 3 - popliteal lymph node, 4 - retropharyngeal lymph node, 5 - tracheobronchial lymph node, 6-mesenteric lymph node

The radiological examination without preparation did not give significant results; this was due to the presence of a very dense layer of connective tissue, this is why the use of iodine-based clouding solution was necessary to properly visualize the content of the organ with its different components.

The image of the x-ray of the axillary lymph node has shown us only that it is normal and typically homogeneous, of density equivalent to that of muscle tissue, the contour is regular, located in a fatty environment.

The maximum diameter in the axial plane is conventionally $15 \mathrm{~cm}$ and $9 \mathrm{~cm}$ for an inguinal lymph node. The size criterion corresponds to our morphometric study data, the inguinal lymph node presented a reniform and oblong image (Fig. 5).

On examination by conventional radiology, which requires the use of iodized product of the dromedary lymph nodes, simultaneously opacifying arteries and veins makes it possible to differentiate from the vascular structures of the organ. It is therefore an essential quality criterion for a well performed examination. After injection, we provided an image of infiltration of contrast product in the internal lymphatic channels, going into the medullary region, in partial improvement, the capsule of the axillary lymph node was lifted for the first time and became opaque and the area surrounding was improved unevenly. Certain peripheral hypo-dense areas of the whole organ that had not improved were observed in the inguinal lymph node. The enhanced contrast imaging also showed that enhancement of contrast occurs mainly in the internal regions, suggesting an initial distribution of contrast of the sub capsular sinuses. Our study showed that most of the internal lymphatic vessels detected were partially and unevenly improved.

We observed that the axillary lymph node is of rounded shape, with a cortex periphery para cortex below including medullary center and hilum normally visible, this image was clear with the change in the shade of opacification. One could also see that the area of extension of the cortical area of the inguinal lymph node is due to a presence probably in the follicles which are very numerous at this level, the appreciation of the invasion of this area at the lower parenchyma leading to a medullary area, many vessels were seen as a fluctuation of the submerged braided folds, the radiological enlarged inguinal lymph node was assessed, the location and the most representative radiographic view. Simultaneous images were also noted and grouped. There was no statistically significant difference in the size of the lymph node between the lateral views of the radiograph, not enlarged and positioned more dorsally, no statistical difference between the right and left lateral, as for the projection on which the lymph node lymphatic was enlarged at a distance, the different compartments seemed well defined (Fig. 6). Visualization of the lymphatic pathways, some of which ran along the cortical area, either down or up to the nodes located at the origin of the hilum or directly at the bottom of the medullary area. Many vessels were seen as a fluctuation of the submerged braided folds, the radiological enlarged inguinal lymph node was assessed, the location and the most representative radiographic view. The other lymphatic vessels reached the periphery of this lymph node. On the left side, we can see lymphatic vessels that also run along the cortical area of the inguinal lymph node with the presence of two lymphatic bags just at the end of the drained area. The lymphatic vessels gradually regress along the sub capsular zone, the spatial organization of the lymphatic channels become opaque, from which they remain independent. The collectors at the level of the axillary lymph node develop first by budding of pre-existing vessels, with several communicating vessels and connect to each other in the lymphatic system.

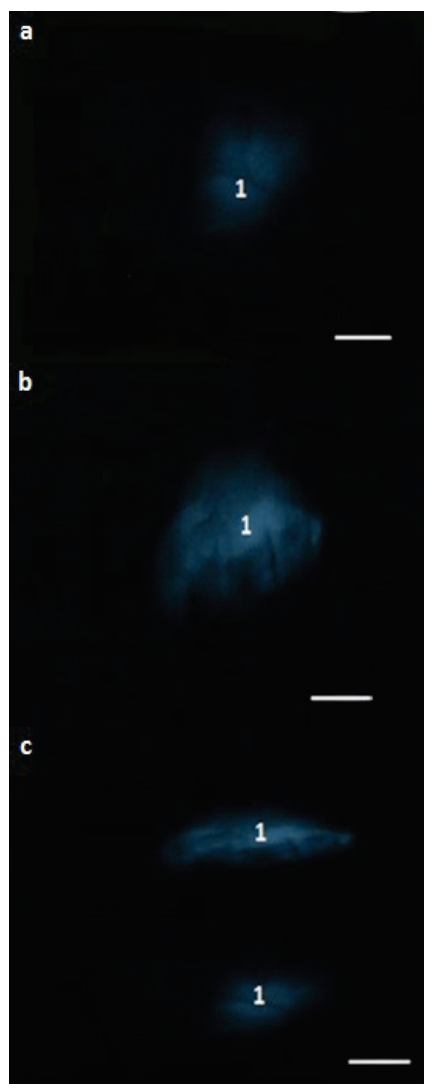

Fig. 5. Standard $\mathrm{x}$-rays without preparation axillary lymph node $(a, b)$, slight opacification in 1 due to the presence of non-significant soft tissue; inguinal lymph node (c), presence of two opacification zones in 1 due to the presence of soft tissue divided into two zones

The CT scanner offers better resolution in contrast and allows us to obtain cross-sections in the three planes of space. The indications in the study of the internal architecture of the axillary lymph node are made by passing through the lateral and posterior regions. The lymph node appears on the first image normal, homogeneous, is typically iso-intense compared to the muscular tissue and hypo-intense compared to the fatty tissue. On the second and third image, the axillary lymph node appears hyper intense compared to the muscle and slightly more intense than fat, that it is isointense and indistinguishable from environmental fat.

Using a CT, we could see photos of the lymph nodes, whose periphery is very clear, which is the capsule, the cortical area is very developed. On the other hand the medullar area was less concentric and this deconcentration goes towards the edges of the hilum. The appearance of two medullar zones in a single lobule, the afferent lymphatic vessel is clearly visible, on the other hand the efferent lymphatic vessels are less visible.

Looking on the second and the third picture, it was noted that the unimproved axillary lymph node had a lower signal intensity than the surrounding fat and appeared homogeneous internally. Also it was observed that the capsule was not very narrow, elongated and the low signal intensi- 
ty was separable from the oval or rounded structures located below this zone whose signal intensity was higher. Theoretically, the histological analysis cites that the rounded structures corresponded to the follicles of the cortical and paracortical zone, while the linear structures corresponded to the medullary sinuses of the lymph node (Fig. 7).

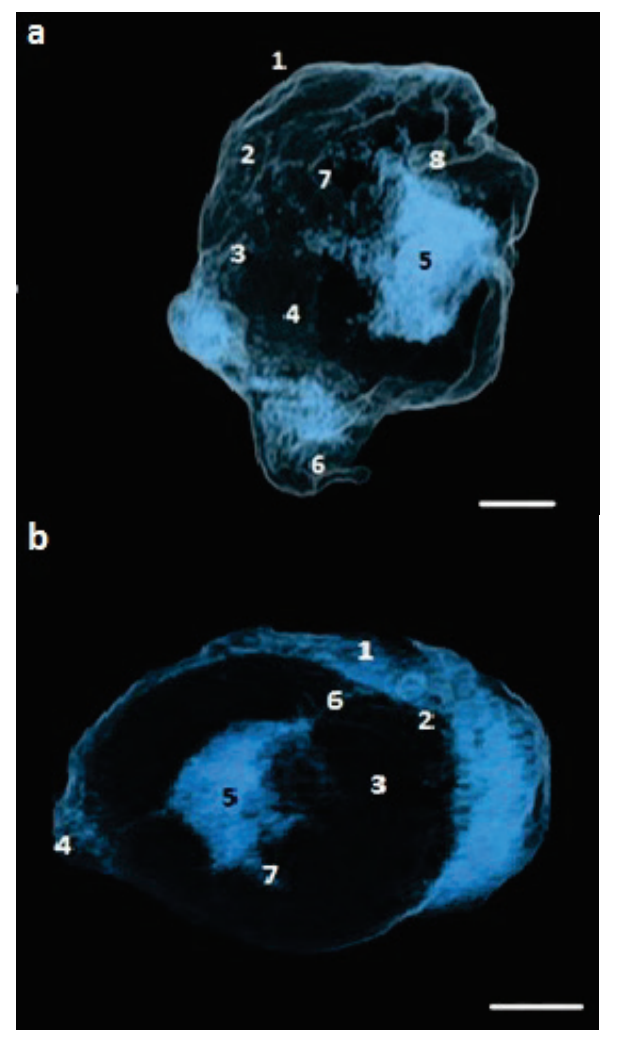

Fig. 6. Conventional radiology with preparation of iodine injection in situ of the lymph node of the dromedary: $a$-axillary lymph node: 1 - capsule,

2 -paracortical zone, 3-subcapsular lymphatic vessels, 4-cortical lymphatic vessels, 5-medullary zone, 6 - gates of the lymph node,

7-zone of the cortex, 8 - peri-medullary lymphatic vessels; $b$-inguinal lymph node: 1 -capsule, 2 - paracortical zones, 3 -cortical zones, 4 -gates of the lymph node, 5 - medullary zones, 6 - cortical lymphatic vessels, 7-peri-medullary lymphatic vessels; bar $-1 \mathrm{~cm}$

A careful examination with the CT scan of a longitudinal section also showed that the cortical area surrounding the medullary area, a difference between the two compartments is clearly seen by a difference in colour, all are wrapped in a capsule, a cross section showed the same results, the lymphatic vessels appear much better, large in size, with tuberosities on the outer surface of the lymph node. At the level of the parenchyma, it has been distinguished that the extension surface of the cortical zone is due to a presence probably in the follicles, which are very numerous at this level, the appreciation of the invasion of this zone in the lower parenchyma going towards the medullary area, simultaneous images were also noted and grouped and which also show that there is no statistically significant difference in the size of the lymph node, not enlarged and positioned more dorsally, no statistical difference between the lateral ones right and left, as for the projection on which the axillary lymph node was enlarged at a distance, the different compartments seemed well defined (Fig. 8).

Further exploration with CT of the inguinal lymph node revealed a slight increase in density, clearly visible on the dorsal sides and that it had more distinct margins on the dorsoventral view due to the anatomical complexity of this area (Fig. 9).

It has also been elucidated that the trabeculae extending centrally from the periphery represent the hilum of the inguinal lymph node and contain, among others, ramifications, it is vertically oriented and has a triangular configuration and extending from the medial cortex until the periphery with a fan-shaped configuration, extending from a delimited central region, the displacement is graduated according to position of the trabecular, it was noted that the medial trabeculae form a misaligned angle.

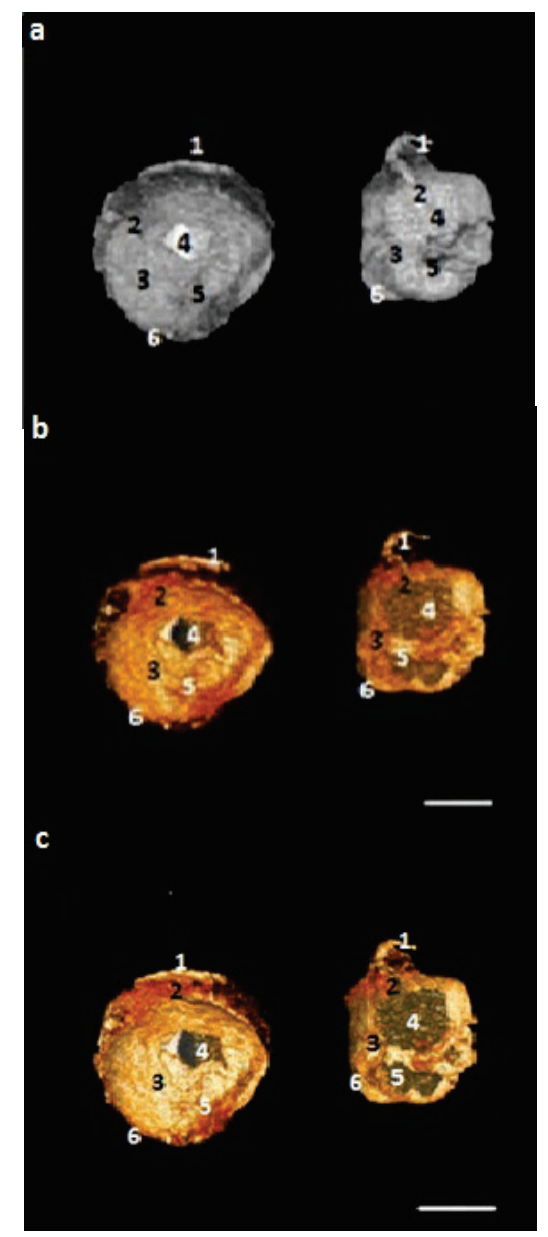

Fig. 7. CT of the axillary lymph node of the dromedary without preparation: $a$-axial view T1 saturation $20 \%, b$-axial view T2 60\% saturation, $c$-axial view T3 85\% saturation: 1 - gates of the lymph node, 2 - paracortical zone, 3 - cortical zone, 4 -medullary zone, 5 -deep medullary zone, 6 -capsule; bar $-1 \mathrm{~cm}$

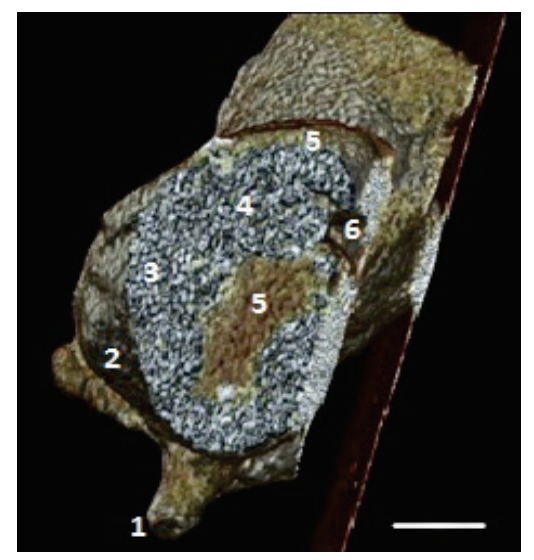

Fig. 8. CT of the dorsoventral inguinal lymph node of the dromedary, T1 saturation 35\%, multi-center view of the different layers of stroma cortical and medullar zone: 1 - gates of the lymph node, 2 - capsule,

3 - cortical zone, 4 - paracortex zone, 5 - medullary zone, 6 -deep cortical zone; bar $-1 \mathrm{~cm}$

\section{Discussion}

Looking at the results we found, we noticed that they are similar to the data of other researchers who studied the morphology and anatomy of the lymph node in animals. The axillary lymph node appears constant, more or less flattened, of almost circular shape, placed at the height of the third dimension on the serrated muscle of the chest and below the axillary vein, its depth is related to the large round muscle and the thoraco-dorsal 
artery (Fowler et al., 1988). The popliteal lymph node appears constant, and has an ovoid shape, is located in the popliteal fossa, on the twin muscles of the leg. The latter is almost on the surface although it is hidden by the thick elastic blade which strengthens the leg fascia. The inguinal lymph node appears bulky, enormous, elongated and fusiform, in the male its location is in the fold of the groin across the rudimentary teats. It rests on the abdominal coat, which has been demonstrated by the author (AbellMagied et al., 2001; Saliman \& Mazher, 2005). The scrotal node is related to the caudal preputial muscles and is limited ventrally by the abdominal subcutaneous vein. While in the female it is located in the inguinal region, the two ganglia are side by side and are in the perimammary position. They are covered by the skin and are related to the pectineal muscles. Topographically, the mesenteric visceral lymph nodes are located in the terminal part of the rectum. Concerning the tracheobronchial lymph node, this lymph node is composed of two symmetrical portions: the right and left tracheobronchial lymph nodes.

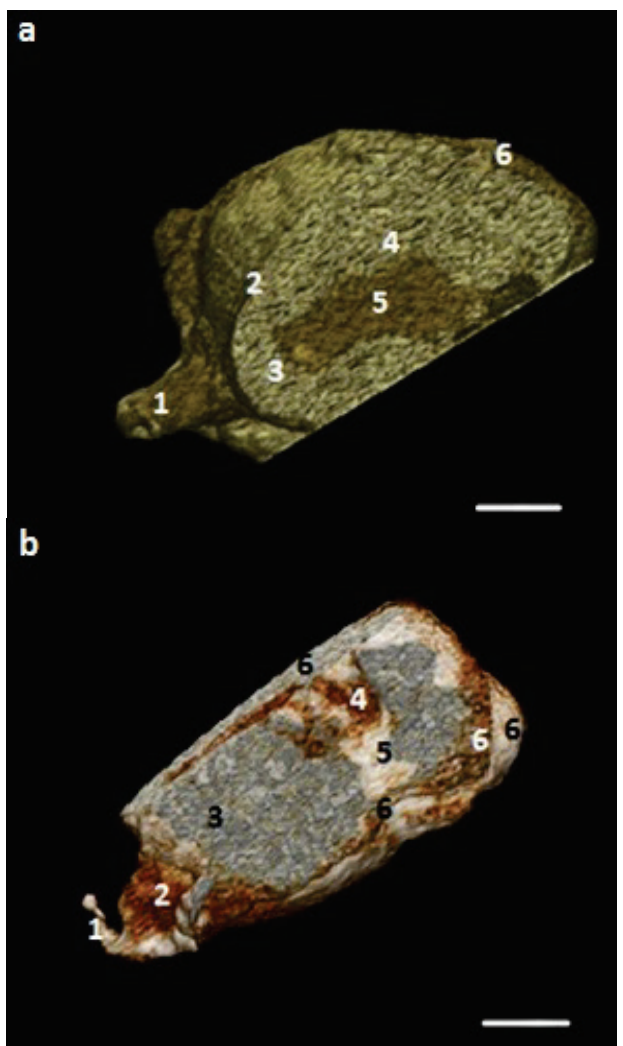

Fig. 9. Short axis oval well limited regular contours with a fatty hilum homogeneous peripheral enhancement with a single lobe containing: $a$-view T1 saturation 35\%: 1 - gates of the lymph node, 2 - capsule,

3 -cortical zone, 4 - paracortical zone, 5 -medullary zone,

6 - subcapsular zone; $b$ - view T1 saturation $85 \%: 1$ - gates of the

lymph node, 2 - subcapsular zone, 3 - cortical zone, 4 - cortical

lymphatic vessels, 5 - medullary zone, 6 - capsular zone; bar $-1 \mathrm{~cm}$

The right tracheobronchial node is placed against the termination of the trachea at the birth of the tracheal bronchus, whereas the left tracheobronchial lymph node was bifurcated with a thin flattened part and the other globose. It is visible on the left behind the aortic arch, for the retropharyngeal lymph node seems bulky and hollowed out by a groove where the common carotid artery lodges; it is in cranial connection with the mandibular gland and dorsally with the occipital artery.

The locations of most of the lymph nodes in different types of animals are largely the same. According to researchers (Gasisova et al., 2017), in cattle and sheep, the nodes are the same, they are bean-shaped, oval or rounded, usually surrounded by fatty tissue and have a grey colour or intense grey in the section.

According to Artemeva \& Chekarova (2019), in goats there is a similar arrangement, but many of them are lunar in shape, also according to the author (Bode et al., 2018) in pigs, many large lymph nodes on the surface have a pronounced lotion or tuberosity (Lossi et al., 2016). Individual lymph nodes are represented by bundles made up of different numbers of small nodules scattered in the fatty tissue (especially in the head and neck). On the cut, the colour of the lymph nodes is pinkish white or white (they are similar to fat, but denser and contact). In horses, the lymph nodes occupy the same areas as in other animals, with minor differences (Janvier et al., 2015). They are made up of groups (20-40) of nodules constituting the packets. The colour is pale grey.

The study of the absolute and relative mass of the lymph nodes carried out by (Gavrilin et al., 2017), in newborn calves revealed that the lymph nodes are characterized by a certain mass and morphometric parameters, which largely depend on the prenatal development of animals, also in calves with a high morpho-functional status of the organism, the absolute mass of the lymph nodes varies in a few grams (Gouda, 2016), which correspond to our results which state the mass of the removed lymph nodes, and calculates the relative mass of each organ with respect to the weight of the animal. It was found that the maximum value of the absolute mass was $42.75 \mathrm{~g}$ on the axillary somatic lymph node, with a relative mass of $0.012 \%$, and the minimum value of absolute mass was $4.2 \mathrm{~g}$ on the tracheobronchial visceral lymph node, with a relative mass of $0.006 \%$. For the other lymph nodes variable results were found, for the inguinal lymph node the absolute mass was $11.5 \mathrm{~g}$ with a relative mass of $0.003 \%$, for the popliteal lymph node the absolute mass noticed was $17.4 \mathrm{~g}$ with a relative mass of $0.005 \%$, for the retro pharyngeal lymph node the absolute mass was $15.7 \mathrm{~g}$, with a relative mass of $0.003 \%$, for the mesenteric lymph node the absolute mass was $7.0 \mathrm{~g}$ with a relative mass of $0.004 \%$.

Regarding the morphometry of the lymph nodes, the length and width of the lymph nodes, the maximum length of $8.2 \mathrm{~cm}$ was found for the axillary somatic lymph node, while the minimum value of $3.9 \mathrm{~cm}$ was found for the tracheobronchial visceral lymph node. For width, the maximum value for width was $6.9 \mathrm{~cm}$ for the axillary somatic lymph node, and the minimum value was $1.3 \mathrm{~cm}$ for the tracheobronchial visceral lymph node, same results found by the authors (Al Aiyan et al., 2019). The study of the lymph node volume revealed to us that the maximum value of the volume was $0.44 \mathrm{~mm}^{3}$ in the axillary somatic lymph node with relative volume of $0.61 \%$, and the minimum value was $0.03 \mathrm{~mm}^{3}$ in the visceral tracheobronchial lymph node with relative volume of $0.08 \%$.

The minimum value for width was $0.9 \mathrm{~cm}$ for the retro-pharyngeal visceral lymph node, data similar to (Abdel-Magied et al., 2001).

Standard radiography without preparation of the organs revealed little data for which the use of contrast agent is necessary because it is a soft organ, as the authors quote (Watson \& Heng, 2017). The images obtained by conventional radiology allowed us to visualize in vitro the internal structure of the dromedary lymph nodes. Using this technique, we have achieved sufficient resolution to reach the micro-vessels of the lymph nodes. To our knowledge, this is the first use of imagery on the dromedary lymph nodes which has provided us with detailed information on the internal morphology of this organ as many researchers have tried to exploit this organ in animals, which is quoted in the review of animals (Stahle et al, 2019).

The resolution imaging capacity in vitro was more beneficial for the study of the lymph node without having it incised by the standard method which involves the elimination of adipose tissue, which could lead to damage to the organ and keep its macroscopic aspect, which could constitute the base of the anatomical structure. Our methods make it possible to visualize the lymphatic vessels. Although the importance of visualizing the different compartments of the lymph node is known, vascularization was a largely unexplored area due to the lack of research.

Several attempts have been made to highlight the intra nodal architecture using the technique of mass infiltration of the anchor dye and histological examination by microscopy to locate the high-endothelial vessels in the lymph node (Gavrilin \& Kolesnyk, 2019). However, this imaging technique can be performed with existing conventional radiology without being limited by the compromise between resolution and depth of penetration. The preparation of the lymph nodes with a contrast agent showed us a thick layer like an envelope penetrating the organ and forming internal lobular cavities, the medullary zones are very clear by the trajectories of the trabecular spans corresponding to the opening of the channels of the 
hilum, the same results were found in the lymph nodes of cattle by researchers (Gadre et al, 2018).

This exploration technique is still being improved to highlight the various anomalies of the lymphatic circulation using non-harmful contrast agents (Davenport et al., 2020). The three-dimensional architecture of the lymph nodes highlighted by computer tomography reveals the presence of segments of the parenchyma formed of a distinct structural unit, surrounded by a layer of connective tissue of variable shape; these results have been cited by the authors (Sun et al., 2019).

The radiological examination without preparation did not give significant results; this is due to the presence of a very dense layer of connective tissue, results similar to the data of (Ying \& Ahuja, 2003).

In our results, we were able to demonstrate that the dromedary lymph nodes, under infiltration of the contrast product in the internal lymphatic channels, the concentration of the product going towards the medullary zone emerges at the level of the hilum by passing through the lymphatic bundle. Regarding CT research, our study had some limitations. The mechanical scanning method used in our study did not offer any infiltration of contrast product, limiting the resolution in the third dimension. This restriction can be overcome by using a two-dimensional matrix network to perform this full three-dimensional imaging. Another limitation of mechanical scanning is the long acquisition time required to obtain sufficient data in each plane. In addition, micro-CT was performed separately on the dromedary lymph node.

By exploring the dromedary lymph nodes by the $\mathrm{CT}$ in vitro, we generally found that the sub capsular periphery is very clear, the cortical zone is very developed, on the other hand the medullary zone is less concentric with the appearance of two medullary areas in a single lobule, the afferent lymphatic vessel is clearly visible, but the efferent lymphatic vessels are less visible.

\section{Conclusion}

In our research, results obtained on the study of the anatomy and topography of the lymph nodes elucidated for the six organs namely; the axillary lymph node appears constant, more or less flattened, almost circular in shape. The popliteal lymph node appears constant, and has an ovoid shape, located in the popliteal fossa, on the twin muscles of the leg, for the inguinal lymph node, it appears large, enormous, elongated and fusiform, it rests on the abdominal coat.

Among the visceral lymph nodes, the mesenteric lymph node is located in the terminal part of the rectum. The tracheobronchial lymph node is superimposed on the bifurcation of the trachea towards the bronchi, while the retro-pharyngeal lymph node seems bulky and hollowed out by a gutter where the common carotid artery lodges. The mass of the lymph nodes studied varies according to the localization of the organ in the satellite defense of the lymphoid system, being of very different length and width.

In our research, we were able to elucidate by conventional radiology the structure and lymphatic circulation with the formation of lobules, all of which are surrounded by a thick layer of connective tissue which is the capsule. The in vitro resolution imaging capacity was more beneficial for the study of the lymph node, without having it incised by the standard method, which involves the elimination of adipose tissue, which could lead to damage to the organ and to maintain its macroscopic appearance, which could constitute the base of the anatomical structure. Our methods make it possible to visualize the lymphatic vessels. Although the importance of visualizing the different compartments of the lymph node is known, vascularization was a largely unexplored area due to the lack of research.

\section{References}

Abdel-Magied, E. M., Taha, A. A. M., Al-Qarawi, A. A., \& Elfaki, M. G. (2001). The parotid, mandibular and lateral retropharyngeal lymph nodes of the camel (Camelus dromedarius). Anatomia, Histologia, Embryologia: Journal of Veterinary Medicine Series C, 30(4), 199-203.

Al Aiyan, A., Abdullah, S., Menon, P., Shawaf, T., Al Darwich, A., \& Barigye, R. (2019). A histologic and histomorphometric study of the second stomach chamber of the dromedary (Camelus dromedarius). Journal of Camel Practice and Research, 26(1), 91.
Artemeva, E., \& Chekarova, I. (2019). Varieties of atypical lymph nodes of water deer (Hydropotes inermis argyropus). IOP Conference Series: Earth and Environmental Science, 403, 012149.

Ashwini, S., Pawar, A. A., Sathisha, K. K., \& Girish, M. H. (2018). Light and electron microscopic study of lungs in Bidri goat and Deccani sheep. The Indian Journal of Veterinary Sciences and Biotechnology, 13(3), 10601.

Bodi, I., H.-Minko, K., Prodan, Z., \& Olah, I. (2020). Compartmentalization of human thymic medulla: facts and hypotheses. Thymus. 145-240.

Davenport, M. S., Perazella, M. A., Yee, J., Dillman, J. R., Fine, D., McDonald, R. J., Rodby, R. A., Wang, C. L., \& Weinreb, J. C. (2020). Use of intravenous iodinated contrast media in patients with kidney disease. Kidney Medicine, 2(1), 85-93.

Elmore, S. A. (2006). Histopathology of the lymph nodes. Toxicologic Pathology, 34(5), 425-454.

Fowler, M. E., Smuts, M. M. S., \& Bezuidenhout, A. J. (1988). Anatomy of the dromedary. The Journal of Zoo Animal Medicine, 19(3), 136.

Gadre, K. M., Ashok, P., Arun, K., \& Mukartal, S. Y. (2018). Comparitive morphometry and histomorphological study on superficial lymph nodes of forelimb and hindlimb in Deccani sheep (Ovies aries) and Bidri goat (Capra hircus). Journal of Experimental Zoology, India, 21(2), 1159-1161.

Gasisova, A. I., Atkenova, A. B., Ahmetzhanova, N. B., Murzabekova, L. M., \& Bekenova, A. C. (2016). Morphostructure of immune system organs in cattle of different age. Anatomia, Histologia, Embryologia, 46(2), 132-142.

Gavrilin, P. M., \& Kolesnyk, A. O. (2019). Lymphatic supply and architectonics of intranodal lymphatic channel of lymph nodes of domestic pig. Theoretical and Applied Veterinary Medicine, 7(3), 158-162.

Gavrilin, P. N., Gavrilina, E. G., \& Evert, V. V. (2017a). Histoarchitectonics of the parenchyma of lymph nodes of mammals with different structure of intranodal lymphatic channel. Ukrainian Journal of Ecology, 7(3), 96-107.

Gavrilin, P. N., Gavrilina, O. G., Brygadyrenko, V. V., \& Rahmoun, D. E. (2017b). Structural and functional units of parenchyma of lymph nodes of dromedaries (Camelus dromedarius). Regulatory Mechanisms in Biosystems, 8(3), 323-332.

Gavrilin, P. N., Lieshchova, M. A., \& Rahmoun, D. E. (2015). Features of zonal structure of the parenchyma and intra-site lymphatic channel of lymph node in dromedary. The Animal Biology, 17(3), 29-37.

Gavrilin, P. N., Lieshchova, M. A., Gavrilina, O. G., \& Boldyreva, T. F. (2018). Prenatal morphogenesis of compartments of the parenchyma of the lymph nodes of domestic cattle (Bos taurus). Regulatory Mechanisms in Biosystems, 9(1), 95-104.

Gor, D. M., Langer, J. E., \& Loevner, L. A. (2006). Imaging of cervical lymph nodes in head and neck cancer: The basics. Radiologic Clinics of North America, 44(1), 101-110.

Gouda, S. (2016). Comparative histomorphology and histochemistry of thoracic aorta in deccani sheep and bidri goat. MOJ Anatomy and Physiology, 2(3), 44.

Harisinghani, M. G. (2012). Pelvic lymph nodes. Atlas of lymph node anatomy, 89-153.

Janvier, V., Evrard, L., Cerri, S., Gougnard, A., \& Busoni, V. (2015). Ultrasonographic findings in 13 horses with lymphoma. Veterinary Radiology and Ultrasound, 57(1), 65-74.

Kelch, I. D., Bogle, G., Sands, G. B., Phillips, A. R. J., LeGrice, I. J., \& Dunbar, P. R. (2019). High-resolution 3D imaging and topological mapping of the lymph node conduit system. PLoS Biology, 17(12), e3000486.

Kraitchman, D., Kamel, I., Weiss, C., \& Georgiades, C. (2017). Elucidation of percutaneously accessible lymph nodes in swine: A large animal model for interventional lymphatic research. Journal of Vascular and Interventional Radiology, 28(3), 451-456.

Lossi, L., D’Angelo, L., De Girolamo, P., \& Merighi, A. (2016). Anatomical features for an adequate choice of experimental animal model in biomedicine: II. Small laboratory rodents, rabbit, and pig. Annals of Anatomy - Anatomischer Anzeiger, 204, 11-28.

Maher, M. A., Farghali, H. A. M., Abdelnaby, E. A., \& Emam, I. A. (2020). Gross anatomical, radiographic and doppler sonographic approach to the infra-auricular parotid region in donkey (Equus asinus). Journal of Equine Veterinary Science, 88, 102968.

Mallet, C., Cornette, R., \& Guadelli, J. (2019). Morphometrical distinction between sheep (Ovis aries) and goat (Capra hircus) using the petrosal bone: Application on french protohistoric sites. International Journal of Osteoarchaeology, 29(4), 525-537.

Margaris, K. N., \& Black, R. A. (2012). Modelling the lymphatic system: Challenges and opportunities. Journal of the Royal Society Interface, 9(69), 601-612.

Mebius, R. E. (2003). Organogenesis of lymphoid tissues. Nature Reviews Immunology, 3(4), 292-303.

Nourinezhad, J., Mazaheri, Y., \& Biglari, Z. (2015). Detailed anatomy of the cranial cervical ganglion in the dromedary camel (Camelus dromedarius). The Anatomical Record, 298(8), 1479-1491. 
Nourinezhad, J., Mazaheri, Y., \& Saberifar, S. (2016). Topography and morphology of the bovine cranial cervical ganglion and its branches. International Journal of Morphology, 34(2), 545-556.

Pabst, R. (2020). The pig as a model for immunology research. Cell and Tissue Research, 380(2), 287-304.

Rebelatto, M. C. (2018). Spleen, lymph nodes, and thymus. Boorman's Pathology of the Rat, 469-491.

Reiter, R., Viehdorfer, M., Hescock, K., Clark, T., \& Nemanic, S. (2017). Effectiveness of a radiographic anatomy software application for enhancing learning of veterinary radiographic anatomy. Journal of Veterinary Medical Education, 2017, 1-9.

Saliman, S. M., \& Mazher, K. M. (2005). Light and electron microscopic studies on some lymph nodes of the adult one-humped camel (Camelus dromedarius). Beni Suef Veterinary Medicine Journal, 15(2), 9-13.

Stahle, J. A., Larson, M. M., Rossmeisl, J. H., Dervisis, N., \& Neelis, D. (2018). Diffusion weighted magnetic resonance imaging is a feasible method for characterizing regional lymph nodes in canine patients with head and neck disease. Veterinary Radiology and Ultrasound, 60(2), 176-183.
Sun, X., Liu, E., Iqbal, A., Wang, T., Wang, X., Haseeb, A., Ahmed, N., Yang, P., \& Chen, Q. (2019). The dynamic distribution of duck Tembusu virus in the spleen of infected shelducks. BMC Veterinary Research, 15, 1.

Taher, E.-S. M., El-Sakhawy, M. A., Moussalam, E.-S., Moussa, M. H. G., \& ElBargeesy, G. (1989). Studies of the lymphoid tissue of lymph nodes of one humped camel fetuses (Camelus dromedarius). Egypt Journal Histology, 12(1), 23-33.

Watson, E., \& Heng, H. G. (2017). Forensic radiology and imaging for veterinary radiologists. Veterinary Radiology and Ultrasound, 58(3), 245-258.

Willard-Mack, C. L. (2006). Normal structure, function, and histology of lymph nodes. Toxicologic Pathology, 34(5), 409-424.

Ying, M., \& Ahuja, A. (2003). Sonography of neck lymph nodes. Part I: Normal lymph nodes. Clinical Radiology, 58(5), 351-358

Zidan, M., \& Pabst, R. (2002). Detection of apoptosis in the lymphoid organs of the dromedary camel. Journal of Camel Practice and Research, 9(2), 163-166.

Zidan, M., \& Pabst, R. (2012). Histological, histochemical and immunohistochemical study of the lymph nodes of the one humped camel (Camelus dromedarius). Veterinary Immunology and Immunopathology, 145(1-2), 191-198. 